Original Research Paper

\title{
The Existing Methods for Motion Control of Flexible Needles along a Curved Path as Part of Robotic Systems for Brachytherapy
}

\author{
${ }^{1}$ Aleksandr Vitaljevich Lopota, \\ ${ }^{1}$ Nikolai Anatoljevich Gryaznov, ${ }^{2}$ Olga Valerjevna Velichko, ${ }^{1}$ Konstantin Yurjevich Senchik, \\ ${ }^{1,2}$ Vyacheslav Valentinovich Kharlamov, ${ }^{1}$ Sergey Alexandrovich Nikitin and ${ }^{1}$ Galina Sergeevna Kireeva \\ ${ }^{I}$ Russian State Scientific Center for Robotics and Technical Cybernetics, St. Petersburg, Russia \\ ${ }^{2}$ Laser Technology Center, St. Petersburg, Russia
}

Article history

Received: 09-11-2015

Revised: 18-01-2016

Accepted: 19-01-2016

Corresponding Author:

Sergey Alexandrovich Nikitin,

Russian State Scientific Center

for Robotics and Technical

Cybernetics, St. Petersburg,

Russia

Email: s.nikitin@rtc.ru

\begin{abstract}
Review of the recent developments in steerable needles and control algorithms of their movements in curvilinear directions in the human body for the modern robotic system to perform brachytherapy in prostate cancer patients is presented. Advantages of steerable needles over the standard ones are described; design aspects of these needles and results of the phantom studies are discussed. Scientific novelty consists in structuring the research information on the newest approaches in the prostate cancer treatment using brachytherapy, about the methods and tools that improve the quality of procedures, reducing time of procedure and making it safer.
\end{abstract}

Keywords: Steerable Needles, Prostate Cancer, Brachytherapy, Robotic System

\section{Introduction}

In 2013 in Russia 31,569 new cases of the prostate cancer were registered with an average annual growth rate of the cancer incidence being 8.09\% (largest for all cancer types in the male population) (Caprin et al., 2015). There are several treatment approaches applicable in prostate cancer patients, which include brachytherapy, hormone therapy, radical prostatectomy and external beam radiotherapy. Brachytherapy is considered as the least invasive method with the possibility of out-patient treatment having a minimum number of treatment sessions. In brachytherapy radioactive seeds are being implanted into the prostate using hollow needles. Depending on the exposure dose there are low-dose and high-dose brachytherapy procedures. Low-dose brachytherapy is used as an independent radical method and today it is one of the leading treatment options for prostate cancer because it allows achieving positive outcomes in patients at low, medium and high risk (Koukourakis et al., 2009; Skowronek, 2013). Generally radioactive seeds implantation is performed manually which provides several drawbacks:

- Relatively small maneuverability because of the fixed pattern

- The complexity of the needle insertion at an angle if required
- Factors of movement and deformation of the prostate and bleeding that occur with needle insertion are not considered in dosimetric planning

- The human-factor impact on the consistency, accuracy and efficiency of the procedure

Therefore there is a growing interest in development and introduction into clinical practice of robotic systems for brachytherapy procedure. Several systems have already been developed, however they have standard rigid needles that do not allow a doctor to avoid certain obstacles during needle introduction and prevent reaching the intended target (Abolhassani et al., 2007a).

\section{Theoretical Background and Literature Review}

It is proven that more than $70 \%$ of tumor foci are located at the periphery of the prostate $(\mathrm{McNeal}$ et al., 1998; Cohen et al., 2008). However, the needle introduction into the peripheral area is challenging puncturing prostate capsule away from central axis, increased needle obliquity angle, inadequacy/non rigidity in supporting of the prostate and single point proximalend actuation of a long slender needle (Nag et al., 2000). Another important factor is the swelling of the prostate during brachytherapy (Kehwar et al., 2011; Nath et al., 
2009). Eapen et al. (2004) discovered that the prostate trauma due to the needle insertion makes a significant contribution to the development of acute urogenital toxicity. The authors proposed to minimize the needle manipulation of the periurethral path. Shah and Ennis (2006) studied the relationship between side effects from the rectum (acute diarrhea) and the number of needles inserted during brachytherapy. More acute diarrhea developed in case of using larger number of needles which apparently was due to the more significant trauma in the anterior wall of the rectum from the needles implanting seeds in the dorsal surface of the prostate. This suggests that reducing the number of needles and the minimal amount of manipulation is one of the factors for improving brachytherapy outcomes.

Problems of needle introduction into the peripheral zone of the prostate can be eliminated by developing a new type of needle that can be easily placed according to the shape of the prostate. Podder et al. (2012) created steerable needles that could 'adjust' under the particular geometry of the prostate (Fig. 1). In the proposed approach needles are inserted near, but not along the central axis of the prostate using curvilinear path (curvilinear approach to radioactive seeds implantation). The studies have shown that using the curvilinear approach improves almost all dosimetric parameters (uniform distribution of radiation dose on the prostate, the mean rectal dose) compared with the standard linear approach. With this approach the insertion area with fewer needles introduced is achieved leading to reduced swelling of the prostate and less discomfort for the patient.

In order to provide the desired parameters for the needles special alloys are being used. As a result, shape memory alloy actuated flexible needles (SMA needles) have been developed. In case of SMA needles tracking the route of the planned needle trajectory is crucial and is hard to achieve during brachytherapy procedures. Ruiz et al. (2012) created robotic system with an SMA needle actuator for the brachytherapy procedure. To track an actual needle position a set point tracking control of a flexible needle actuated by SMA using electromagnetic sensory feedback is performed. Preliminary results revealed that the SMA actuator may be adequate for creating required curvature for geometrically conforming the prostate gland.

Wood et al. (2010) used a curvilinear approach in clinical practice to introduce steerable needles in transcutaneous medical interventions on the kidney. Orlando et al. (2015) compared the adequacy of three methods to control the introduction of SMA needles which were electromagnetic sensors, direct visualization (web camera) and ultrasound. The standard deviation of the values from the planned trajectory was minimal in case of electromagnetic sensors, which made the authors to consider this method of feedback as the most accurate. The worst results were registered in case of ultrasound use: Poor image quality compared to a web camera requires more computing for pattern matching path of the needle.

Considering the possibility of using steerable needles, special attention should be paid to their design. In planning low-dose brachytherapy delivery and positioning of radioactive seeds according to the preoperative dosimetric plan is one of the most important stages of the procedure. Therefore, the relationship between the needle design and the method of its motion in the tissue influences the significance of tissue damage, the ability to avoid obstacles along a curvilinear path and positioning accuracy of the seeds. Ko et al. (2011) developed a flexible probe potentially capable of threedimensional steering in soft tissue inspired by natureovipositor of a Giant Ichneumon wasp (Fig. 2).

In experimental studies such needles were moved with different levels of bias (Fig. 3). Positioning accuracy of the needle tip was $0.68 \pm 1.45 \mathrm{~mm}$.

Swaney et al. (2013) proposed to improve the controllability of a needle and to increase the curvature of the trajectory by giving a degree of freedom to asymmetrical tip of the needle placing nitinol wires along the needle (Fig. 4). A degree of freedom of the tip allows increasing the angle of deviation, therefore increasing maneuverability of the needle and its controllability resulting in potentially less tissue damage.
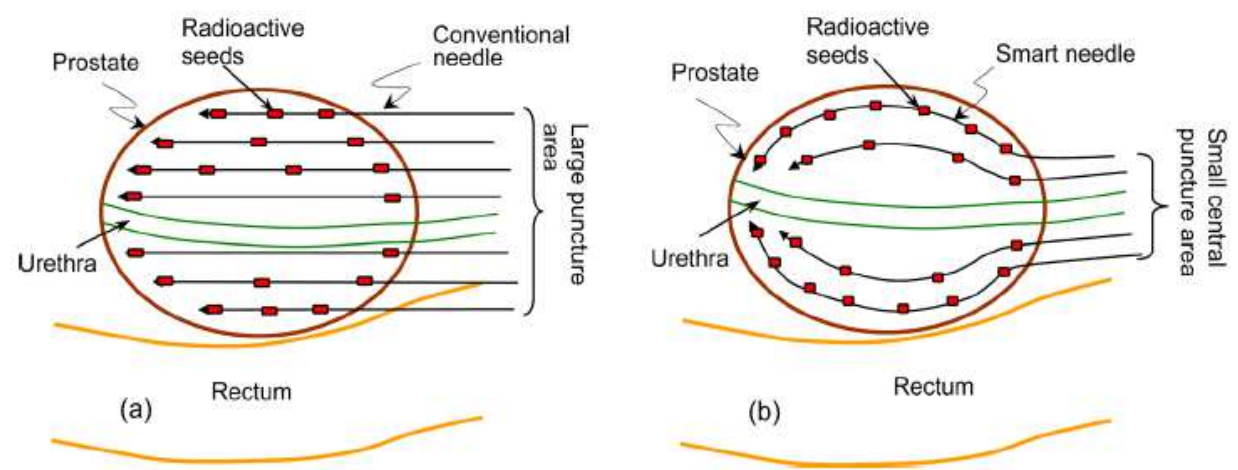

Fig. 1. Accessing various parts of the prostate-(a) conventional rectilinear approach of prostate brachytherapy needle insertion pattern with straight needles requiring 7 needles and (b) proposed curvilinear conformal smart needle insertion requiring 4 needles (in any convenient needle entry orientation) (Podder et al., 2012) 


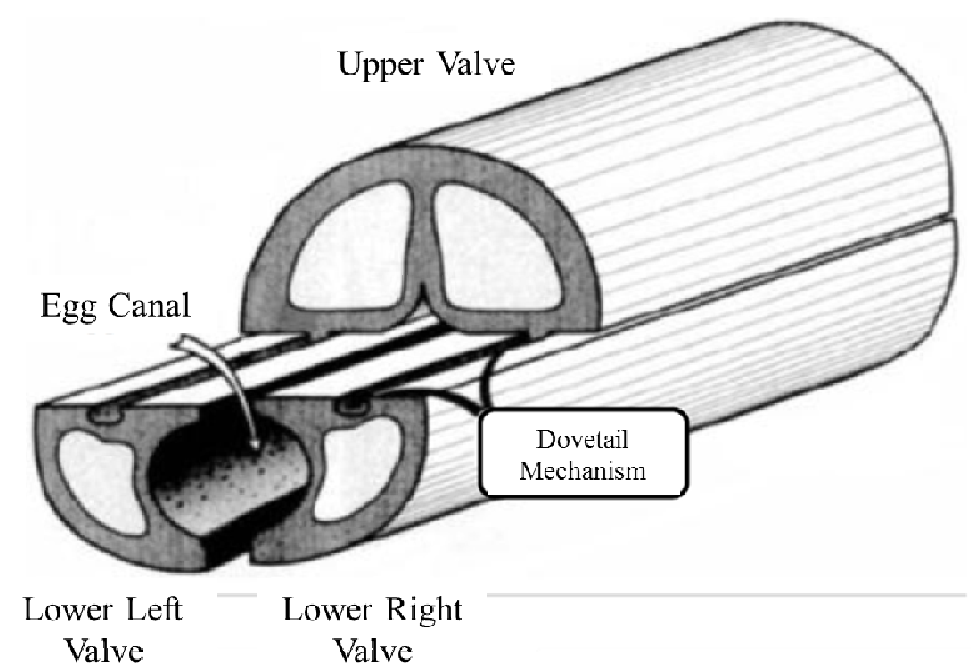

Fig. 2. Diagrammatic representation of oblique view of transversely cut ovipositor (Ko et al., 2011)

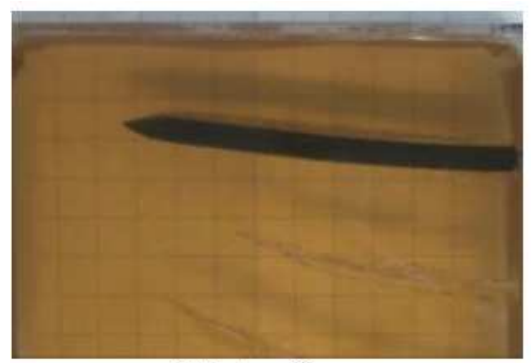

Offset $=0 \mathrm{~mm}$

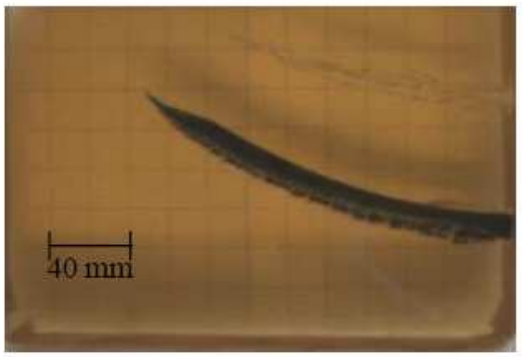

Offset $=-15 \mathrm{~mm}$

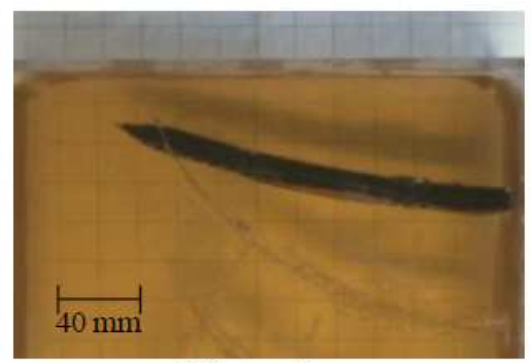

Offset $=-5 \mathrm{~mm}$

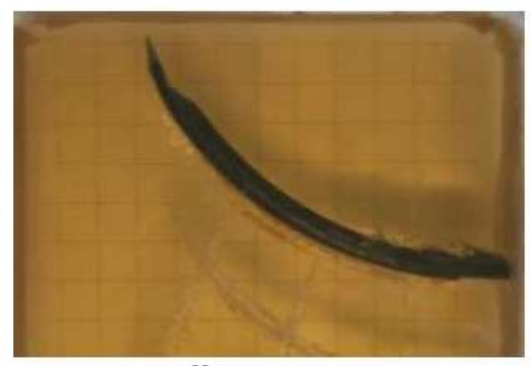

Offset $=-25 \mathrm{~mm}$

Fig. 3. Captured flexible probe trajectories for different steering offset values (Ko et al., 2011)

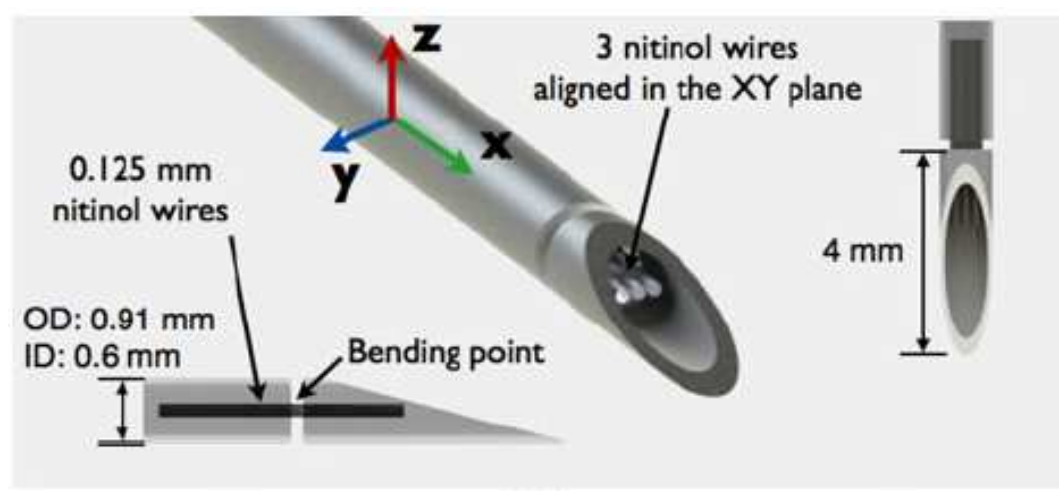

Fig. 4. Flexure-tip needle design. The nitinol wires that comprise the flexure joint bend at the gap between the needle shaft and the tip as forces are applied to the bevel tip by tissue (Swaney et al., 2013) 


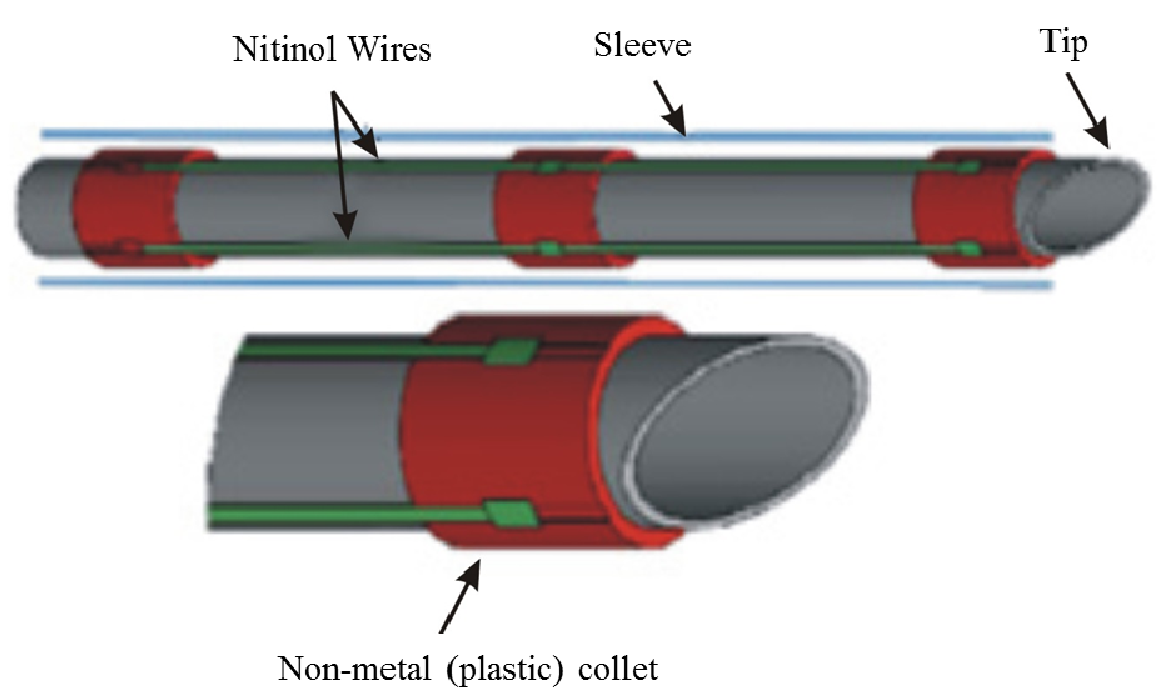

Fig. 5. The longitudinal body segment smart needle design (Podder et al., 2010)

Podder et al. (2010) proposed another construction of a steerable needle with nitinol wires on its outer side in the special clamping sleeve (Fig. 5). This construction provides a direct contact of the tissue with wires which have the property of shape memory with a temperature increase.

To create a robotic system for brachytherapy it is important not only to develop special needles and optimal insertion trajectory, but also to establish the methods to control the needle behavior in the body during brachytherapy. Over the past 20 years, several attempts to control the steerable needles have been made. Abolhassani et al. (2007b) proposed a model for the control of a needle deflection from a target using ultrasound guidance as a feedback. Maghsoudi and Jahed (2013) presented an option of a needle control by estimating the force exerted to the needle at subcutaneous interventions. Ko et al. (2011) described closed loop control system driven using an electromagnetic sensor which tracks the path of the needles in the 2D mode. Kallem and Cowan (2009) developed an alignment control algorithm for inserting needles along the planar trajectory. Using a stereo camera to determine the position of the needle tip, the authors implemented the concept of 'observer' for the approximate calculation of parameters such as the rotational degree of freedom of the needle, which was previously impossible to measure. Deflection of a needle with a tapered end of targeted trajectory can be monitored using a special algorithm which allows varying the curvature of the needle, changing the ratio between the period of the needle rotation and the total period of insertion (Engh et al., 2006; Minhas et al., 2007). Glozman and Shoham (2004) used inverse kinematics of a controlled needle inserted into the viscoelastic model of the tissue. The authors have shown that the needle trajectory is not unique, the introduction and optimization of insertion process can be accomplished by minimizing the lateral pressure of the needle on the tissue. Dehghan and Salcudean (2009) optimized the angle and depth of needle insertion, using a 3D tissue model including several 'targets'.

Abayazid et al. (2014) conducted an interesting experimental study where they used the $3 \mathrm{D}$ algorithms for planning and control of the needle trajectory under ultrasound guidance, which remains one of the most affordable imaging techniques in the clinical practice. Needle insertion procedure using proposed robotic control system is shown in Fig. 6. The anatomical regions of interest in the patient are acquired preoperatively using ultrasound images. Based on the images, the clinician identifies the target location and sensitive structures, such as glands or blood vessels and other obstacles, such as bones. The path planning algorithm generates a needle trajectory to avoid obstacles and reach the target. The planner generates new paths intra-operatively based on the updated needle tip position (obtained from ultrasound images) and target position during insertion. The needle insertion procedure is autonomous under supervision of the clinician.

The experimental setup was studied in soft tissue phantom made of gelatin mixture. The silicon powder was added to the mixture to create acoustic scattering same as occurs in human tissues. SMA needles were used made of nickel and titanium alloy. The authors considered several experimental scenarios in order to assess the behavior and capabilities of the proposed algorithms for tracking the insertion route, path planning and needle insertion control (Fig. 7). 


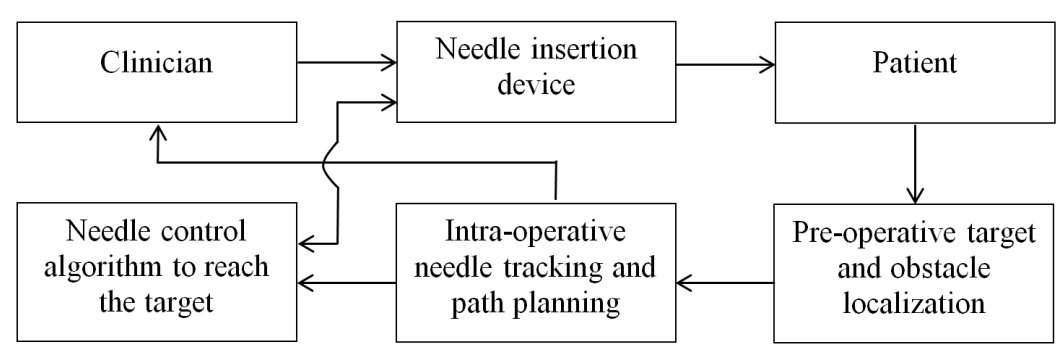

Fig. 6. The workflow presents a clinically viable robotic needle steering system. The needle insertion device controls the direction of insertion inside the patient's soft tissue. Needle tip tracking and path planning are performed intra-operatively to provide control algorithm and the clinician with data required to control the insertion device (Abayazid et al., 2014)

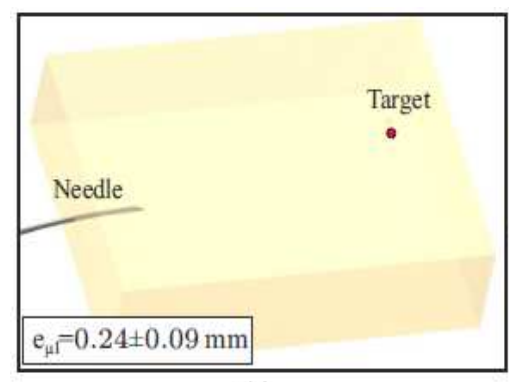

(a)

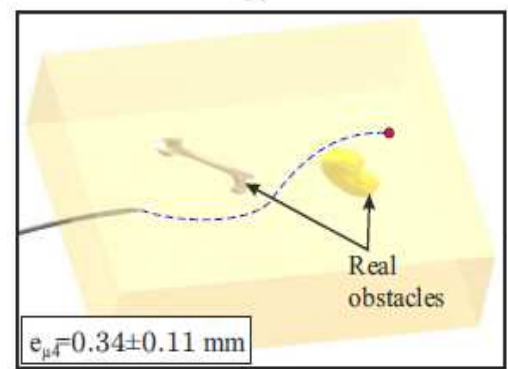

(d)

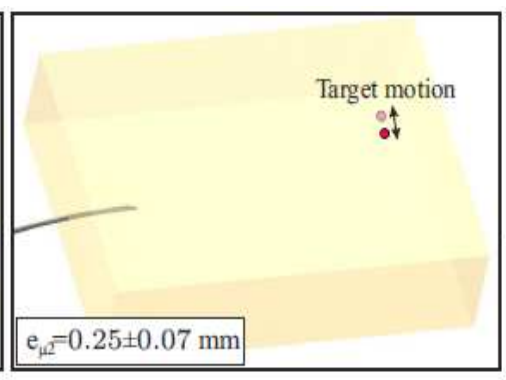

(b)

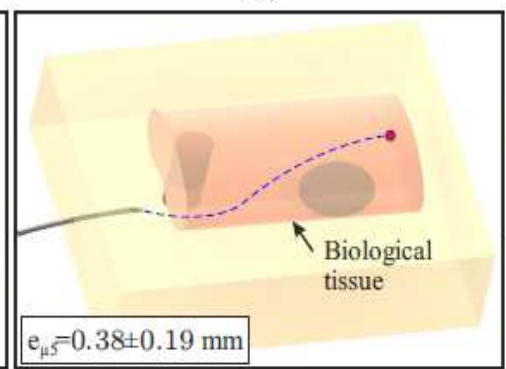

(e)

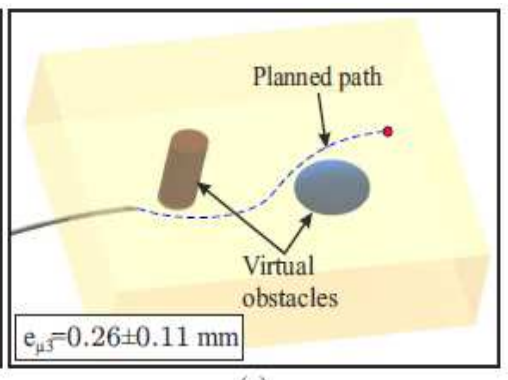

(c)

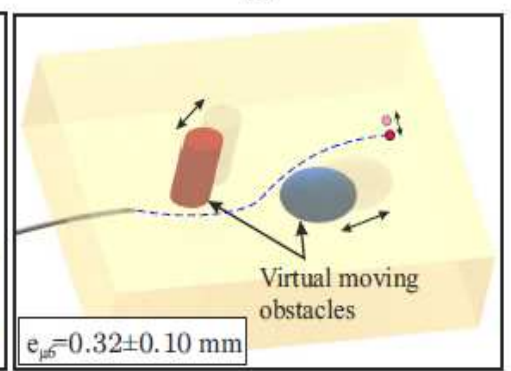

(f)

Fig. 7. The experimental cases (Abayazid et al., 2014)

For each of the cases under consideration the targeting error $\left(e_{\mu}\right)$ was calculated, which was determined as an absolute distance between the actual target needle tip position and its pre-set position obtained from the needle tracking algorithm. The main advantage of these algorithms is that the needle is rotated only when necessary to change the direction of insertion. Accordingly, the reduced number of needle rotations potentially reduces trauma to the soft tissues. The authors note that the needle visibility in ultrasound images is deteriorated due to shadows surrounding the solid obstacles during insertion and this affects the targeting accuracy. The targeting error increases while needle steering in biological tissue due to the tissue in homogeneity. The experimental results show that the mean targeting error ranges from $0.24 \pm 0.09$ to $0.38 \pm 0.19$ $\mathrm{mm}$. However in clinical studies, more variables are expected that may reduce the targeting accuracy such as physiological motion, fluid flow and tissue in homogeneity.
The steering system can be extended to detect the patient movements that occur during needle insertion such as respiration and fluid flow. In general, it was shown in experimental settings that the proposed algorithms for the robotic needle insertion system under the ultrasound guidance provides sufficient precision needle insertion and can reduce the trauma on biological tissues.

\section{Results}

According to the authors, the steerable needle described in (Ko et al., 2011) is moved owing to the steering off set, which in turn only depends on the forward motion of each segment. The proposed control approach is expected to cause less strain to the surrounding tissue and, in case of successful prototype miniaturization, less tissue damage.

Steerable needle, which is described in (Swaney et al., 2013) was made by using surgical needles with standard dimensions. These needles required no miniaturization. 
Questions remain about the damage of the tissue which is associated with the fact that a needle with asymmetrical steerable tip was used. According to the authors, it is necessary to conduct some additional research on the impact of the needle on the tissue when implementing curved paths.

Steerable needle, which is described in (Podder et al., 2010), has the same principle as in (Swaney et al., 2013). The main difference in the location of nitinol wires is that they are located on the outside. Overall, this study indicated that clinical implementation of the proposed steerable needle may potentially improve radiation dose distribution and reduce dose to critical organs and thereby would potentially improve quality of life and survival of the prostate cancer patients.

\section{Discussion of the Results}

Various data were presented on implementing curvilinear approach in brachytherapy procedure using steerable needles and motion control. It is important to note that in gelatin phantoms physiological processes taking place in the real tissue cannot be considered. The tissue can swell, increasing in volume and move affected by inserted needles. The motion adjustment of the needle and prostate in real time by using ultrasound scanners is required. We can conclude that future research in this field will need additional experiments and clinical trials to make sure that technology, tools and control algorithms are working well.

\section{Conclusion and Further Research}

Currently at the stage of theoretical and practical advance the scientific and technological groundwork is being laid in the field of steerable needles and methods of control over their movements as a part of robotic brachytherapy systems. The presented approaches have a few differences such as principles of action, the design, accuracy and extent of tissue damage.

Particular attention is paid to the development of simulating and control systems of the needle movement on a curved path by using ultrasound scanners and MRI. The optimization of control algorithms, increasing positioning accuracy and reducing errors remain the major issues at this stage.

\section{Acknowledgement}

The authors would like to express their gratitude to the management of the Central Research Institute of Robotics and Cybernetics for their support.

\section{Funding Information}

This article was prepared with the financial support from the Ministry of Education and Science of Russian
Federation for the research under the Agreement of 27.06.2014, grant 14.575.21.0035 (unique identifier RFMEFI57514X0035) for the implementation of the federal target program "Research and development on priority directions of scientific-technological complex of Russia for 2014-2020 years".

\section{Author's Contributions}

Aleksandr Vitaljevich Lopota: Organized the study, writing the manuscript.

Nikolai Anatoljevich Gryaznov: Was engaged in checking information, writing the manuscript.

Olga Valerjevna Velichko: Was engaged in checking information, writing the manuscript.

Konstantin Yurjevich Senchik: Contributed in development of conceptual framework, results analysis, drafting of the article.

Vyacheslav Valentinovich Kharlamov: Was responsible for data collecting and analysis of the results.

Sergey Alexandrovich Nikitin: Participated in data collecting, writing and editing of the manuscript.

Galina Sergeevna Kireeva: Contributed in data collecting, writing and editing of the manuscript.

\section{Ethics}

The authors have no conflicts of interest in the development of the research and publication of this article.

\section{References}

Abayazid, M., G.J. Vrooijink, S. Patil, R. Alterovitz and S. Misra, 2014. Experimental evaluation of ultrasound-guided 3D needle steering in biological tissue. Int. J. CARS, 9: 931-939.

DOI: $10.1007 / \mathrm{s} 11548-014-0987-y$

Abolhassani, N., R. Patel and F. Ayazi, 2007a. Needle control along desired tracks in robotic prostate brachytherapy. Proceedings of the IEEE International Conference on Systems, Man and Cybernetics, Oct. 7-10, IEEE Xplore Press, Montreal, Que., pp: 1361-1366. DOI: 10.1109/ICSMC.2007.4413819

Abolhassani, N., R.V. Patel and M. Moallem, 2007b. Needle insertion into soft tissue: A survey. Med. Eng. Phys., 29: 413-431.

DOI: 10.1016/j.medengphy.2006.07.003

Caprin, A.D., V.V. Starinsky and G.V. Petrova, 2015. Malignancies in Russia in 2013 (morbidity and mortality). P. Herzen Moscow Oncology Research Institute, Moscow, pp: 249.

Cohen, R.J., B.A. Shannon, M. Phillips, R.E. Moorin and T.M. Wheeler et al., 2008. Central zone carcinoma of the prostate gland: A distinct tumor type with poor prognostic features. J. Urol., 179: 1762-1767. DOI: 10.1016/j.juro.2008.01.017 
Dehghan, E. and S.E. Salcudean, 2009. Needle insertion parameter optimization for brachytherapy. IEEE Trans. Robotics, 25: 303-315. DOI: $10.1109 /$ TRO.2008.2011415

Eapen, L., C. Kayser, Y. Deshaies, G. Perry and E. Choan et al., 2004. Correlating the degree of needle trauma during prostate brachytherapy and the development of acute urinary toxicity. Int. J. Radiat. Oncol. Biol. Phys., 59: 1392-1394.

DOI: 10.1016/j.ijrobp.2004.01.041

Engh, J.A., G. Podnar, S.Y. Khoo and C.N. Riviere, 2006. Flexible needle steering system for percutaneous access to deep zones of the brain. Proceedings of the IEEE 32nd Annual Northeast Bioengineering Conference, Apr. 01-02, IEEE Xplore Press, Easton, pp: 103-104.

DOI: 10.1109/NEBC.2006.1629773

Glozman, D. and M. Shoham, 2004. Flexible needle steering and optimal trajectory planning for percutaneous therapies. Proceedings of the International Conference on Medical Image Computing and Computer-Assisted Intervention, Sept. 26-29, Saint-Malo, France, pp: 137-144. DOI: $10.1007 / 978-3-540-30136-3 \quad 18$

Kallem, V. and N.J. Cowan, 2009. Image guidance of flexible tip-steerable needles. IEEE Trans. Robot., 25: 191-196. DOI: 10.1109/TRO.2008.2010357

Kehwar, T.S., H.A. Jones, M.S. Huq and R.P. Smith, 2011. Influence of prostatic edema on ${ }^{131} \mathrm{Cs}$ permanent prostate seed implants: A dosimetric and radiobiological study. Int. J. Radiat Oncol. Biol. Phys., 80: 621-627.

DOI: $10.1016 /$ j.ijrobp.2010.07.001

Ko, S.Y., F. Luca and F. Baena, 2011. Closed-loop planar motion control of a steerable probe with a "programmable bevel" inspired by nature. IEEE Trans. Robot., 27: 970-983.

DOI: 10.1109/TRO.2011.2159411

Koukourakis, G., N. Kelekis and V. Armonis 2009. Brachytherapy for prostate cancer: A systematic review. Adv. Urol. DOI: 10.1155/2009/327945

Maghsoudi, A. and M. Jahed, 2013. Model-based needle control in prostate percutaneous procedures. Proc. Inst. Mech. Eng. H., 227: 58-71. DOI: $10.1177 / 0954411912458489$

McNeal, J.E., E.A. Redwine, F.S. Freiha and T.A. Stamey, 1988. Zonal distribution of prostatic adenocarcinoma: Correlation with histologic pattern and direction of spread. Am. J. Surg. Pathol., 12: 897-906. DOI: 10.1097/00000478-198812000-00001

Minhas, D.S., J.A. Engh, M.M. Fenske and C.N. Riviere, 2007. Modeling of needle steering via duty-cycled spinning. Proceedings of the IEEE 29th Annual International Conference of the IEEE Engineering in Medicine and Biology Society, Aug. 22-26, IEEE Xplore Press, Lyon, pp: 2756-2759.

DOI: 10.1109/IEMBS.2007.4352899
Nag, S., W. Bice and K. DeWyngaert, B. Prestidge and R. Stock et al., 2000. The American brachytherapy society recommendations for permanent prostate brachytherapy postimplant dosimetric analysis. Int. J. Radiat. Oncol, Biol, Phys., 46: 221-230. DOI: 10.1016/S0360-3016(99)00351-X

Nath, R., W.S. Bice, W.M. Butler, Z. Chen and A.S. Meigooni et al., 2009. AAPM recommendations on dose prescription and reporting methods for permanent interstitial brachytherapy for prostate cancer: Report of task group 137. Med. Phys., 36: 5310-5322. DOI: 10.1118/1.3246613

Orlando, F.M.J., K. Madhukar, K. Franz, B. Konh and P. Hutapea et al., 2015. Control of shape memory alloy actuated flexible needle using multimodal sensory feedbacks. J. Automat. Control Eng., 3: 428-434.

Podder, T.K., A.P. Dicker, P. Hutapea, K. Darvish and Y. $\mathrm{Yu}$ et al., 2012. A novel curvilinear approach for prostate seed implantation. Med. Phys., 39: 1887-1892. DOI: 10.1118/1.3694110

Podder, T.K., P. Hutapea, K. Darvish, A. Dicker and Y. Yu et al., 2010. Smart needling system for fully conformal radiation dose delivery in treating prostate cancer. ASME Conference on Smart Materials, Adaptive Structures and Intelligent Systems, (SIS' 10), pp: 893-896. DOI: 10.1115/SMASIS2010-3919

Ruiz, B., P. Hutapea and K. Darvish, 2012. SMA actuated flexible needle control using EM sensor feedback for prostate brachytherapy. Proceedings of the IEEE International Conference on Robotic and Automation Needle Steering Workshop.

Shah, J.N. and R.D. Ennis, 2006. Rectal toxicity profile after transperineal interstitial permanent prostate brachytherapy: Use of a comprehensive toxicity scoring system and identification of rectal dosimetric toxicity predictors. Int. J. Radiat. Oncol. Biol. Phys., 64: 817-824.

DOI: $10.1016 /$ j.ijrobp.2005.08.042

Skowronek, J., 2013. Low-dose-rate or high-dose-rate brachytherapy in treatment of prostate cancerbetween options. Contemp Brachytherapy, 5: 33-41. DOI: $10.5114 /$ jcb.2013.34342

Swaney, P.J., J. Burgner and H.B. Gilbert, 2013. A flexure-based steerable needle: High curvature with reduced tissue damage. IEEE Trans Biomed Eng., 60: 906-909. DOI: 10.1109/TBME.2012.2230001

Wood, N.A., K. Shahrour and M.C. Ost, 2010. Needle steering system using duty-cycled rotation for percutaneous kidney access. Proceedings of the Annual International Conference of the IEEE Engineering in Medicine and Biology Society, Aug. 31-Sept. 4, IEEE Xplore Press, Buenos Aires, pp: 267-272. DOI: 10.1109/IEMBS.2010.5626514 